\title{
DEGRADACIÓN FOTOCATALÍTICA DEL COLORANTE AMARILLO REACTIVO 145 EMPLEANDO ESTRUCTURAS DE Fe(III)-TiO, SINTETIZADAS DEL MINERAL ILMENITA
}

\section{PHOTOCATALYTIC DEGRADATION OF REACTIVE YELLOW 145 USING Fe(III)-TiO 2 STRUCTURES SYNTHESIZED FROM ILMENITE}

\author{
Juan Alberto TORRES-LUNA, José Gregorio CARRIAZO* \\ Grupo Estado Sólido y Catálisis Ambiental (ESCA), Departamento de Química, Facultad de \\ Ciencias, Universidad Nacional de Colombia, Bogotá. \\ *Autor Corresponsal. E-mail: jcarriazog@unal.edu.co
}

Historia del Artículo

Recibido: Julio 13, 2016

Evaluado: Enero 06, 2017

Aceptado: Enero 23, 2017

Disponible: Febrero 07, 2017

Resumen |

En el presente trabajo se llevó a cabo la degradación (decoloración) fotocatalítica heterogénea del colorante textil "amarillo reactivo 145" en medio acuoso, empleando un conjunto de sólidos de dióxido de titanio dopado con $\mathrm{Fe}^{3+}, \mathrm{Fe}(\mathrm{III})-\mathrm{TiO}_{2}$, sintetizados a partir del mineral natural ilmenita mediante extracción de especies de titanio-hierro en medio ácido a diferentes temperaturas. Los sólidos se caracterizaron por fluorescencia de rayos X, difracción de rayos X, adsorción de nitrógeno y espectroscopía IR. En todos los casos se halló una estructura de anatasa sustituida con $\mathrm{Fe}^{3+}$, mesoporosa y de área superficial muy importante. Los materiales sintetizados presentaron actividad catalítica elevada en la degradación fotoasistida (con radiación UV) del colorante textil, alcanzando porcentajes de conversión iguales o mayores que $85 \%$, con baja potencia de irradiación (0,4 watts) y condiciones suaves de reacción (presión atmosférica y $25^{\circ} \mathrm{C}$ ).

Palabras Clave: Amarillo reactivo 145, degradación de colorantes textiles, $\mathrm{TiO}_{2}$, dióxido de titanio dopado con hierro (III).

\section{Abstract}

In this work, the heterogeneous photocatalytic degradation (decolorization) of the textile dye "reactive yellow 145 " in aqueous solution, using a set of $\mathrm{Fe}^{3+}$-doped titanium dioxide solids, $\mathrm{Fe}(\mathrm{III})-\mathrm{TiO}_{2}$, was carried out. The catalysts were synthesized from ilmenite (a natural inorganic material) by extraction of titanium-iron species in acidic solution at different temperatures. The solids were characterized by X-ray fluorescence, X-ray powder diffraction, nitrogen adsorption, and IR spectroscopy. In all cases, mesoporous $\mathrm{Fe}^{3+}$-substituted anatase structures with remarkable surface areas were found. The synthesized materials showed high catalytic activity in the photo-assisted degradation (using UV-radiation) of the textile dye, reaching conversion percentages equal to or higher than $85 \%$, with low light power (0,4 watts) and mild condition (atmospheric pressure and $25^{\circ} \mathrm{C}$ ).

Keywords: Reactive yellow 145, degradation of textile dyes, $\mathrm{TiO}_{2}$, iron(III)-doped titanium dioxide.

\section{INTRODUCCIÓN |}

La actividad industrial actual y el desarrollo de la vida moderna en la sociedad contemporánea traen consigo la generación de residuos químicos de fábrica y de tipo doméstico. Dentro de la gran diversidad de residuos industriales, los compuestos orgánicos disueltos en medios acuosos representan un riesgo real para la conservación de los ecosistemas debido a que muchos de ellos son considerablemente tóxicos, algunos alteran el metabolismo de las especies presentes y en general todos aumentan la demanda química de oxígeno y afectan el pH. Los colorantes textiles constituyen un tipo de compuestos químicos de enorme preocupación ambiental debido a su impacto negativo sobre los cuerpos de aguas naturales; son tóxicos, algunos mutagénicos y carcinogénicos (Torres-Luna et al., 2016) y tienen altos coeficientes de absortividad molar, por lo que pequeñas cantidades de estos compuestos son capaces de modificar fuertemente la coloración y reducir la penetración de la luz en los cuerpos de aguas naturales, alterando el equilibrio ecológico en dichos ecosistemas. 
La industria textil representa un importante sector económico a nivel mundial. En Colombia, ha jugado un papel determinante en el desarrollo del país, contribuyendo al crecimiento económico, a las exportaciones y a la generación de empleo por más de 80 años (Ministerio del Medio Ambiente, 2001). La cadena textil y de confecciones es la responsable del $12,2 \%$ de la producción industrial y cerca del $6 \%$ de las exportaciones totales (DAMA, 2004), aportando en un $8 \%$ del PIB manufacturero y en un $3 \%$ del PIB nacional (Crediseguro, 2010). Dichas cifras denotan la importancia estratégica de este sector en la economía Colombiana, cuyas operaciones industriales se ubican principalmente en las ciudades de Medellín y Bogotá.

Los efluentes textiles tienen una composición química compleja, debido a la presencia de una amplia variedad de colorantes (Ministerio del Medio Ambiente, 2001) y otros productos químicos, convirtiéndose en un desafío ambiental (Oller et al., 2011). La composición química de dichos efluentes puede variar dependiendo de los procesos, la materia prima y la maquinaria empleada (Oller et al., 2011). Las características típicas de las aguas residuales de la industria textil pueden ser resumidas por su valor de Demanda Química de Oxígeno (DQO) en el intervalo de 150 a $12000 \mathrm{mg} / \mathrm{L}$, la cantidad de sólidos totales en suspensión entre 2900 y $3100 \mathrm{mg} / \mathrm{L}$ y la Demanda Bioquímica de Oxígeno (DBO) en el intervalo de 80 a $6000 \mathrm{mg} / \mathrm{L}$ (Oller et al., 2011). Se estima que, a nivel mundial, se producen anualmente más de 700000 toneladas de aproximadamente 10000 tipos de colorantes textiles disponibles comercialmente (Robinson et al., 2001; Azbar et al., 2004; Verma et al., 2012), de los cuales un buen porcentaje de ellos se pierde, es vertido a las aguas residuales (Patil y Shukla, 2015), durante el proceso de teñido (Tabla 1).

Tabla 1. Niveles de fijación de algunos colorantes sobre fibras especificas

\begin{tabular}{cccc}
\multicolumn{4}{c}{ (Zaharia y Suteu, 2012) } \\
\hline $\begin{array}{c}\text { Clases de } \\
\text { colorantes }\end{array}$ & Fibra & $\begin{array}{c}\text { Nivel de fijación } \\
(\%)\end{array}$ & Pérdida (\%) \\
\hline Ácidos & Poliamida & $80-95$ & $5-20$ \\
Básicos & Acrílico & $95-100$ & $0-5$ \\
Directos & Celulosa & $70-95$ & $5-30$ \\
Dispersos & Poliéster & $90-100$ & $0-10$ \\
Reactivos & Celulosa & $50-90$ & $10-50$ \\
A la tina & Celulosa & $80-95$ & $5-20$ \\
\hline
\end{tabular}

Las moléculas de los colorantes textiles usados en la actualidad son de estructuras muy variadas y complejas, la mayoría de ellos son de origen sintético y muy soluble en agua. Debido a su estructura química, son altamente resistentes a la degradación por exposición a la luz, al agua y a muchas sustancias químicas (Robinson et al., 2001; Azbar et al., 2004). Dentro de las diferentes clases de colorantes, los azoicos son ampliamente usados en la industria textil. Éstos se caracterizan por contener uno o más dobles enlaces nitrógeno-nitrógeno $(-\mathrm{N}=\mathrm{N}-$ ) en su estructura química y actualmente representan cerca del $50 \%$ del mercado mundial (De Souza et al., 2010). El colorante conocido como amarillo reactivo 145 (AR145) es un colorante azoico, altamente soluble en agua, se considera aniónico en solución acuosa, contiene el grupo monoclorotriazina en su estructura (Figura 1) y es ampliamente usado en la industria textil para el teñido de algodón (Gül y Özcan-Yıldırım, 2009; Patil y Shukla, 2015). Los colorantes azoicos son difíciles de retirar totalmente por métodos convencionales (adsorción, coagulación, métodos biológicos, filtración con membranas, entre otros), ya que estas metodologías no alcanzan a destruir completamente dichos contaminantes en concentraciones bajas (Gül y Özcan-Yıldırım, 2009; Khataee, 2009). Los procesos avanzados de oxidación (PAOs), son una serie de metodologías de oxidación que se basan en la generación de especies muy reactivas, entre las que se destaca el radical hidroxilo $(\bullet \mathrm{OH})$, el cual es un potente agente oxidante, capaz de degradar una amplia variedad de contaminantes orgánicos en medio acuoso, de forma rápida y no selectiva (Gül y Özcan-Yıldırım, 2009, Bokare y Choi, 2014, Muñoz et al., 2015).

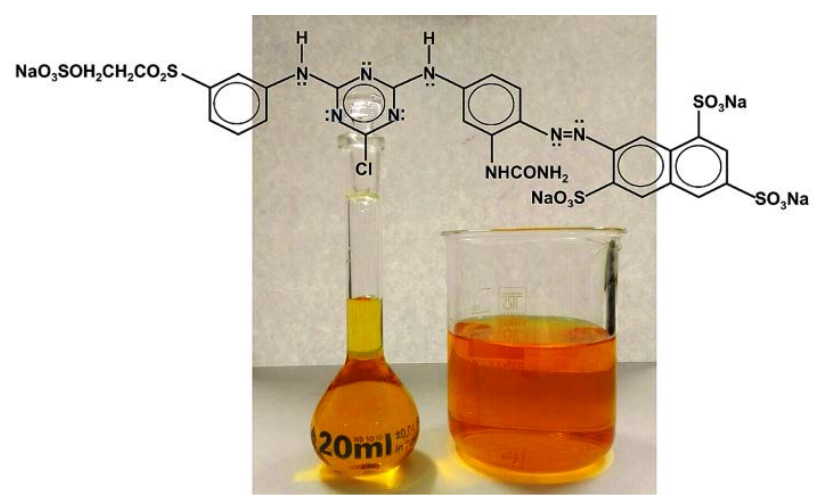

Figura 1. Estructura de la molécula del colorante amarillo reactivo 145 (AR145). $\mathrm{C}_{28} \mathrm{H}_{20} \mathrm{ClN}_{9} \mathrm{Na}_{4} \mathrm{O}_{16} \mathrm{~S}_{5}, 1026.25 \mathrm{~g} / \mathrm{mol}$, CAS: 93050-80-7.

Dentro de los PAOs se destaca la fotocatálisis heterogénea. La degradación fotocatalítica heterogénea de colorantes textiles se considera un método eficiente bajo condiciones de temperatura y presión ambientales, e involucra comúnmente la irradiación del medio de reacción con luz UV $(\lambda<400 \mathrm{~nm})$ en presencia de un semiconductor (fotocatalizador) apropiado (Torres-Luna, et al., 2016). El fotocatalizador más usado es el $\mathrm{TiO}_{2}$, principalmente en su forma de anatasa, y su eficiencia aumenta cuando se le dopa con iones de metales de transición, como el hierro $\left(\mathrm{Fe}^{3+}\right)$. Muchos estudios muestran que la incorporación de iones $\mathrm{Fe}^{3+}$ en la estructura de anatasa aumenta la actividad de dicho sólido en procesos fotocatalíticos, porque dicha incorporación disminuye el "band-gap" (Zhang et al., 2006; Teoh et al., 2007; Carriazo et al., 2010). Por otra parte, las propiedades texturales del $\mathrm{TiO}_{2}$ juegan un papel importante en su actividad catalítica, encontrándose que las estructuras mesoporosas con alta área superficial aumentan el número y la densidad de sitios de reacción y ofrecen mayor estabilidad térmica que las nanopartículas de $\mathrm{TiO}_{2}$ convencionales (Torres-Luna et al., 2014). Pero las propiedades texturales del $\mathrm{TiO}_{2}$, al igual que la cristalinidad y la fase cristalográfica deseada dependen del método de síntesis (Torres-Luna et 
al., 2014); por ello, en el presente trabajo se empleó la síntesis de dióxido de titanio mesoporoso dopado con iones $\mathrm{Fe}^{3+}, \mathrm{Fe}(\mathrm{III})-\mathrm{TiO}_{2}$, a través de una ruta de síntesis directa, establecida con éxito en trabajos anteriores (Torres-Luna et al., 2014). En dicha síntesis, se emplea el mineral ilmenita como fuente natural de titanio y de hierro, sin la necesidad de utilizar reactivos adicionales para controlar la mesoporosidad del nuevo material. La ilmenita $\left(\mathrm{FeTiO}_{3} \mathrm{O}\right.$ $\mathrm{FeO} \bullet \mathrm{TiO}_{2}$ ) es un mineral de estructura hexagonal que se encuentra en algunas rocas ígneas y en arenas negras de playas, es fuente principal de $\mathrm{TiO}_{2}$ a nivel industrial y constituye reservas importantes en el territorio colombiano.

El presente trabajo detalla el estudio de la decoloración (degradación oxidativa) fotocatalizada del colorante amarillo reactivo 145 (AR145), ampliamente usado en la industria textil. En dicha reacción se pone a prueba un conjunto de sólidos cuya estructura consiste en dióxido de titanio dopado con $\mathrm{Fe}^{3+}\left(\mathrm{Fe}(\mathrm{III})-\mathrm{TiO}_{2}\right)$, sintetizados por vía directa a partir del mineral ilmenita (Torres-Luna et al., 2014). Algunos trabajos anteriores han estudiado la decoloración de colorantes azoicos con catalizadores comerciales, con catalizadores sintetizados mediante precursores de costo elevado o con sistemas más complejos (Sathishkumar et al., 2011; Colpini et al., 2014; Patil y Shukla, 2015; Guimaraes de Oliveira et al., 2016); pero según nuestro conocimiento, el presente trabajo es innovador al emplear estructuras fotocatalíticamente activas, extraídas del mineral natural ilmenita, en la decoloración eficiente del colorante azoico AR145.

\section{MATERIALES Y MÉTODOS}

\section{Materiales de partida}

Se empleó como materia prima el mineral ilmenita proveniente de Mitú, departamento de Vaupés-Colombia. Dicho mineral fue suministrado por el Servicio Geológico Colombiano (antes Ingeominas). El mineral natural se purificó, mediante separación magnética, retirando los residuos de arena y otros contaminantes. En las reacciones de síntesis se utilizó ácido sulfúrico (Merck, 98\%), hierro en polvo (J. T. Baker) e hidróxido de sodio (Mallinckrodt, 99,0\%). El colorante textil AR145 $\left(\mathrm{C}_{28} \mathrm{H}_{20} \mathrm{CIN}_{9} \mathrm{Na}_{4} \mathrm{O}_{16} \mathrm{~S}_{5}, 1026.25\right.$ g/mol, CAS: 93050-80-7, Ciba-Geigy) fue suministrado por una empresa local (Bogotá) y utilizado sin purificación adicional. Se empleó $\mathrm{TiO}_{2}$ Degussa P25 (Evonik, $50 \mathrm{~m}^{2} / \mathrm{g}$, tamaño de partícula $\approx 25 \mathrm{~nm}$ ) como fotocatalizador comercial de referencia y $\mathrm{H}_{2} \mathrm{O}_{2}$ (Riedel-de Haën, 35\%).

\section{Síntesis de los sólidos}

Se realizó la síntesis de una serie de sólidos mediante la extracción de especies polioxocatiónicas de hierro-titanio del mineral de partida (ilmenita), usando concentraciones de $\mathrm{H}_{2} \mathrm{SO}_{4}$ y temperatura inferiores a las empleadas comercialmente en el proceso del sulfato. Para ello, se realizaron ensayos usando una masa constante de ilmenita de $18,0 \mathrm{~g}$ y un volumen de $32,0 \mathrm{~mL}$ de ácido sulfúrico a diferentes concentraciones: $20 \%, 50 \%$ y $80 \%$. De igual forma, se varió la temperatura de reacción con tres valores diferentes: $50{ }^{\circ} \mathrm{C}, 70{ }^{\circ} \mathrm{C}$ y $110^{\circ} \mathrm{C}$. El tiempo del proceso de extracción fue de 90 minutos. El proceso de extracción de las especies de titanio-hierro se llevó a cabo en un balón de tres bocas. El montaje permite adicionar los reactivos y disponer de un sistema de reflujo con trampa de gases, así como también controlar la temperatura interna del sistema de reacción, para lo cual se utilizó una plancha de calentamiento con sensor de temperatura. La reacción involucrada en el proceso de extracción produce oxisulfato de titanio y sulfato de hierro(II) como subproducto (Chernet, 1999; Sasikumar et al., 2004):

$$
\mathrm{FeTiO}_{3}+2 \mathrm{H}_{2} \mathrm{SO}_{4} \rightarrow \mathrm{TiOSO}_{4}+\mathrm{FeSO}_{4}+2 \mathrm{H}_{2} \mathrm{O}
$$

La mayor parte de los iones férricos $\left(\mathrm{Fe}^{3+}\right)$ generados durante el proceso fueron reducidos a iones ferrosos $\left(\mathrm{Fe}^{2+}\right)$ mediante la adición de un exceso de Fe (metálico) en polvo, luego la solución resultante se enfrió a $10{ }^{\circ} \mathrm{C}$, para posteriormente retirar el sulfato de hierro en forma de cristales de sulfato ferroso heptahidratado, $\mathrm{Fe}_{2} \mathrm{SO}_{4} \bullet 7 \mathrm{H}_{2} \mathrm{O}$ (Chernet, 1999; Sasikumar et al., 2004). Los residuos sólidos fueron retirados por decantación/filtración. Una fracción pequeña de iones $\mathrm{Fe}^{3+}$ de la solución queda incorporada en la estructura de los polihidroxicationes de titanio. La disolución remanente, así obtenida (disolución con especies polihidroxicatiónicas de hierro-titanio, $\left[\mathrm{Fe}_{\mathrm{x}} \mathrm{Ti}_{(8-}\right.$ $\left.{ }_{x)} \mathrm{O}_{8}(\mathrm{OH})_{12}\left(\mathrm{H}_{2} \mathrm{O}\right)_{m}\right]^{\mathrm{nt}}$, donde $\left.\mathrm{n}=4-\mathrm{x}\right)$, se le ajustó el $\mathrm{pH}$ a 1,2 con solución ( $1 \mathrm{M}$ ) de hidróxido de sodio y posteriormente se sometió a un proceso de hidrólisis bajo reflujo, durante una hora a presión atmosférica. Los sólidos resultantes fueron lavados con agua desionizada, secados a $60^{\circ} \mathrm{C}$ y calcinados a $400{ }^{\circ} \mathrm{C}$, durante dos horas en atmósfera estática de aire. Estos materiales fueron triturados y tamizados en malla ASTM número 100. Los nuevos materiales obtenidos corresponden a las especies de óxidos de titanio dopado con $\mathrm{Fe}^{3+}$ (Torres-Luna et al., 2014) y se denotan (P\%ácidotemperatura) de acuerdo a las condiciones de extracción de las especies de Fe-Ti. Por ejemplo, P50-70 y P50-110 son los sólidos (en polvo) preparados empleando una solución de ácido sulfúrico al $50 \%$ y temperaturas de 70 ó $110{ }^{\circ} \mathrm{C}$, respectivamente.

\section{Caracterización de los sólidos}

El análisis químico elemental de los catalizadores se realizó mediante la técnica de fluorescencia de rayos X (FRX), usando un espectrómetro Philips Magix Pro PW-2440 equipado con un tubo de rodio y una potencia máxima de 4 kW. El análisis por difracción de rayos $X$ en polvo (DRX) se desarrolló en un equipo Panalytical X'Pert PRO MPD, con ánodo de $\mathrm{Cu}$ (radiación Ka, $\lambda=1,54056 \AA$ ). Los difractogramas se tomaron a temperatura ambiente, con monocromador, tamaño de paso de $0,02{ }^{\circ} 2 \theta$ y tiempo de paso $10 \mathrm{~s}$. Las propiedades texturales de los sólidos se determinaron mediante isotermas de adsorción de nitrógeno tomadas a 77 $\mathrm{K}$, usando un quipo Micromeritics ASAP 2020 en el intervalo 
de presiones relativas $\left(\mathrm{P} / \mathrm{P}_{0}\right)$ de $1 \times 10^{-5}$ a 0,99 . Las muestras se desgasificaron previamente a $350{ }^{\circ} \mathrm{C}$ por 4 horas. Los tamaños de poros se determinaron por el método BJH. Los espectros IR se tomaron en un equipo NICOLET Thermo Scientific iS10, haciendo pastillas con $\mathrm{KBr}$ ( $2 \mathrm{mg}$ de muestra en $200 \mathrm{mg}$ de $\mathrm{KBr}$ ) en el intervalo de número de onda de 400 a $4000 \mathrm{~cm}^{-1}$.

\section{Ensayos catalíticos}

Se puso a prueba el desempeño fotocatalítico de los sólidos sintetizados, $\mathrm{Fe}(\mathrm{III})-\mathrm{TiO}_{2}$, en la oxidación del colorante comercial amarillo reactivo 145 (AR145) en medio acuoso diluido. Las disoluciones acuosas del colorante fueron preparadas con agua desionizada y sometidas a ultrasonido durante 10 minutos con el objeto de acelerar el proceso de disolución. El espectro ultravioleta-visible (UVVis) del colorante (Figura 2a) muestra la longitud de onda de máxima absorción en el espectro visible (418 nm). Las reacciones fueron seguidas mediante la cuantificación del colorante en este valor de longitud de onda, es decir mediante la desaparición de la señal correspondiente $(\lambda=418$ $\mathrm{nm}$ ), usando un espectrofotómetro UV/vis Jenway 7305.

La degradación fotocatalítica del colorante en solución se llevó a cabo en un reactor de vidrio tipo semi-batch (totalmente cubierto con papel aluminio), con capacidad de $1000 \mathrm{~mL}$, abierto a la atmósfera, termostatado a $25^{\circ} \mathrm{C}$, con agitación constante (500 rpm) y con un electrodo acoplado (potenciómetro Milwaukee MW 102) para registrar constantemente las variaciones de $\mathrm{pH}$ del medio de reacción (Figura 2b). Asimismo, el reactor estuvo provisto de una lámpara comercial de luz UV (intensidad máxima entre 330 y $390 \mathrm{~nm}$ ) con potencia real de 0,4 W, separada del medio de reacción por un tubo de cuarzo. Adicionalmente, el reactor fue conectado a un dosificador automático (bomba peristáltica Masterflex C/L modelo 77120-62) y a un compresor de aire, por medio de los cuales se logró la adición continúa de una disolución de peróxido de hidrógeno y de un flujo de aire filtrado, respectivamente (Figura 2b).

La concentración del colorante durante el transcurso de la reacción se determinó a partir de alícuotas $(1 \mathrm{~mL}$ de muestra, pasada por filtro Millipore $0,45 \mu \mathrm{m}$ ), tomadas a intervalos de tiempo y empleando una curva de calibración que relaciona la absorbancia de la muestra con la concentración de la misma (Ley de Lambert-Beer). El intervalo de concentraciones en dicha curva fue de 0,5 a 40 $m g L^{-1}$, con un coeficiente de correlación, $R^{2}=0,999$. El límite de detección fue $L D=0,11$ ppm y el límite de cuantificación $\mathrm{LC}=0,40 \mathrm{ppm}$. Previo a los ensayos catalíticos se estableció el tiempo de equilibrio adsorción-desorción del colorante sobre los catalizadores (6o minutos), para lo cual se siguió igualmente la desaparición de la señal del colorante por espectrofotometría (estos ensayos se hicieron a $25{ }^{\circ} \mathrm{C}, 500$ rpm, en ausencia de luz y del oxidante- $\mathrm{H}_{2} \mathrm{O}_{2}$ /aire) hasta que su concentración en la disolución fuera constante. (a)

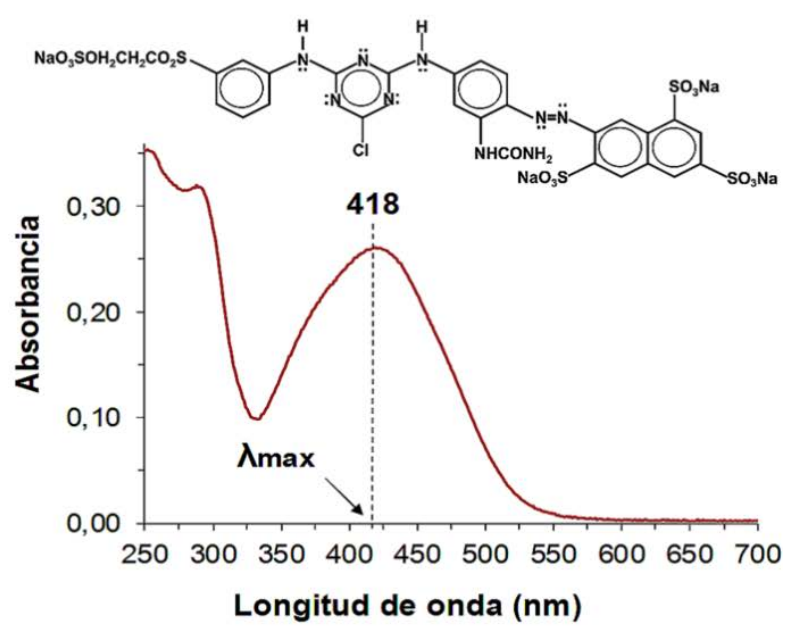

(b)

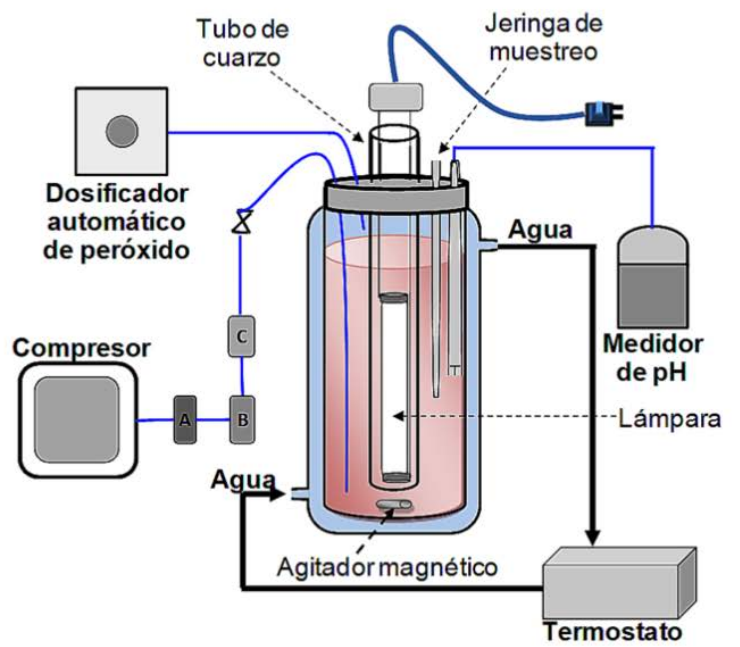

Figura 2. a) Espectro UV-Vis (experimental) del colorante amarillo reactivo 145 (AR145). b) Esquema del reactor empleado en la oxidación fotocatalítica del colorante AR145. A, B y C son filtros que permiten retirar partículas de polvo, agua y $\mathrm{CO}_{2}$ presentes en el flujo de aire, respectivamente.

Para evaluar el proceso de adsorción, se emplearon 100 $\mathrm{mL}$ de una disolución de AR145 (20 $\left.\mathrm{mg} \mathrm{L}^{-1}\right)$ en contacto directo con $20 \mathrm{mg}$ de catalizador (200 ppm de catalizador), a diferentes valores de $\mathrm{pH}$. El $\mathrm{pH}$ de la mezcla se ajustó con pequeñas gotas de ácido sulfúrico $1 \mathrm{M} \circ \mathrm{NaOH} 1 \mathrm{M}$. El proceso fotocatalítico (reacción) se inicia (encendiendo de la lámpara, activando el flujo de aire y de $\mathrm{H}_{2} \mathrm{O}_{2}$ ) una vez se ha llegado al equilibrio dinámico de adsorción-desorción; tiempo en el cual se toma una muestra, a la que se le denomina muestra de concentración a tiempo cero $\left(C_{0}\right)$. Los porcentajes de degradación (\%D), o de conversión, del colorante se calcularon con la siguiente ecuación: 


$$
\% D=\left(\frac{C_{0}-C_{t}}{C_{0}}\right) \times 100
$$

Dónde $C_{0}$ es la concentración inicial del colorante (mg. $\mathrm{L}^{-}$ $\left.{ }^{1}\right)$ y $C_{t}$ es la concentración del colorante $\left(\mathrm{mg} \cdot \mathrm{L}^{-1}\right)$ a un tiempo $t$ de la reacción.

Para cada ensayo catalítico, el reactor se cargó con 650 $\mathrm{mL}$ de una solución de AR145 $20 \mathrm{mg} \cdot \mathrm{L}^{-1}\left(1,95 \times 10^{-5} \mathrm{M}\right)$ con agitación constante de $500 \mathrm{rpm}$ y se adicionó una solución de $\mathrm{H}_{2} \mathrm{O}_{2}\left(0,3 \mathrm{M}\right.$; con un flujo de $2 \mathrm{~mL} \cdot \mathrm{h}^{-1}$, durante $5 \mathrm{~h}$ ) como agente oxidante. La cantidad de peróxido adicionado se ajustó de acuerdo a la estequiometría de la reacción. La temperatura de reacción fue de $25^{\circ} \mathrm{C} \pm 1{ }^{\circ} \mathrm{C}$. Inicialmente se estudió la variación de los valores de $\mathrm{pH}(3,6 ; 5,6 ; 7,0$ y 9,0), del flujo de aire y de la carga de catalizador, con el objeto de encontrar las condiciones óptimas de la reacción, para lo cual se seleccionó uno de los sólidos sintetizados.

\section{RESULTADOS Y DISCUSIÓN}

\section{Caracterización de los sólidos}

El análisis químico por fluorescencia de rayos $\mathrm{X}$, indicó la siguiente composición elemental para la ilmenita natural (mineral de partida): $27,52 \%$ de Ti, 34,61\% de Fe, 0,7\% de Si y $0,32 \%$ de $\mathrm{Al}$, confirmando los mayores contenidos de hierro y titanio. Es necesario resaltar que no se ha expresado la composición como óxidos, sino como elementos simples. Por lo tanto, gran parte de la masa restante de mineral es oxígeno. Además, las pequeñas cantidades de silicio y aluminio evidencian la presencia de pequeñas cantidades de contaminantes, posiblemente aluminosilicatos. La Tabla 2 muestra los contenidos de Fe y Ti en los sólidos sintetizados, revelando el elevado contenido de titanio en los mismos. Estos resultados indican que la cantidad de titanio extraído y de hierro incorporado en los sólidos es dependiente de las condiciones de extracción.

Tabla 2. Contenidos de hierro y titanio en los sólidos sintetizados

\begin{tabular}{ccc} 
Sólidos & Fe (\%) & Ti (\%) \\
\hline P80-50 & 5,45 & 41,96 \\
P50-50 & 2,85 & 39,47 \\
P20-50 & 0,37 & 49,34 \\
P80-70 & 2,57 & 44,16 \\
P50-70 & 0,99 & 42,49 \\
P20-70 & 0,30 & 53,65 \\
P80-110 & 0,50 & 57,00 \\
P50-110 & 0,24 & 57,67 \\
P20-110 & 0,71 & 46,99 \\
\hline
\end{tabular}

La Figura 3 muestra el difractograma del mineral ilmenita, donde se observan las señales características del titanato de hierro $\left(\mathrm{FeTiO}_{3}\right){ }^{\circ} 2 \theta=23,8 ; 32,5 ; 35,2 ; 48,7 ;$ y 53,9 (Ganesh et al., 2012, García-Muñoz et al., 2016, Torres-Luna et al., 2016). Además, en esta misma figura, se encuentran los perfiles de difracción de los sólidos sinterizados. En ellos se observa claramente que la estructura obtenida corresponde a $\mathrm{TiO}_{2}$ en su fase cristalográfica anatasa $\left({ }^{\circ} 2 \theta=25,3 ; 37,8\right.$; $48,1 ; 54,4)$. No se observan señales propias de óxidos de hierro, a pesar de que el análisis químico confirmó la presencia de hierro en cada uno de los sólidos, lo que permite suponer una posible sustitución isomórfica de $\mathrm{Fe}^{3+}$ en el dióxido de titanio, o quizá la formación de óxidos de hierro amorfos. Sin embargo, en estudios anteriores sobre este tipo de sólidos (Carriazo et al., 2010; Sathishkumar et al., 2011, Torres-Luna et al., 2014), se ha discutido ampliamente la substitución isomórfica y se ha establecido la incorporación de $\mathrm{Fe}^{3+}$ en la red cristalina de $\mathrm{TiO}_{2}$. En la Figura 3 también se muestra el perfil de difracción de rayos $X$ (tomado en el presente trabajo) del $\mathrm{TiO}_{2}$ comercial (Evonik P25, tomado como referencia), el cual presenta las fases anatasa y rutilo $\left({ }^{\circ} 2 \theta=27,5 ; 36,2\right)$. Anatasa es la fase de dióxido de titanio mayoritariamente empleada en fotocatálisis, por lo que los resultados de difracción de rayos $\mathrm{X}$ confirman la síntesis exitosa de fotocatalizadores de $\mathrm{TiO}_{2}$ dopados con $\mathrm{Fe}^{3+}$, $\mathrm{Fe}(\mathrm{III})-\mathrm{TiO}_{2}$, como se ha establecido en trabajos previos (Carriazo et al., 2010; Torres-Luna et al., 2014).

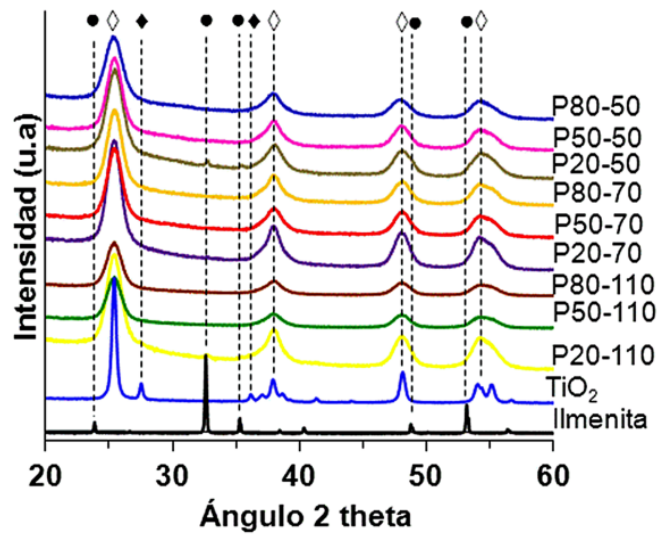

Figura 3. Patrones de difracción de rayos $X$ de la ilmenita, de los sólidos sintetizados y del $\mathrm{TiO}_{2}$ comercial (Evonik P25) usado como referencia (• ilmenita, $\diamond$ anatasa, $\bullet$ rutilo).

Los sólidos sintetizados mostraron mejores propiedades texturales que el $\mathrm{TiO}_{2}$ de referencia (Tabla 3), revelando su gran potencialidad como catalizadores mesoporosos (diámetro de poro alrededor de $7 \mathrm{~nm}$ ) de área superficial elevada (176 a $213 \mathrm{~m}^{2} / \mathrm{g}$ ). Estos aspectos son de gran importancia en catálisis heterogénea, toda vez que facilitan la interacción y el tráfico molecular de las especies involucradas en la reacción química.

Tabla 3. Propiedades texturales (área superficial y tamaño de poro) de los sólidos sintetizados.

\begin{tabular}{ccc}
\multicolumn{3}{c}{ sólidos sintetizados. } \\
\hline Sólido & Área BET $\left(\mathbf{m}^{2} \mathbf{g}^{-1}\right)$ & Radio promedio de poro $(\mathbf{n m})$ \\
\hline P80-50 & 213 & 3,4 \\
P50-50 & 192 & 3,4 \\
P20-50 & 200 & 3,4 \\
P80-70 & 176 & 3,4 \\
P50-70 & - & - \\
P20-70 & - & - \\
P80-110 & 187 & 3,4 \\
P50-110 & 192 & 3,4 \\
P20-110 & 186 & 4,1 \\
$\mathrm{TiO}_{2}$ Evonik & 55 & - \\
\hline
\end{tabular}




\section{Degradación del colorante amarillo reactivo 145 (AR145)}

La reacción química balanceada de la degradación del colorante AR145, asumiendo una mineralización completa, es:

$$
\begin{aligned}
& \mathrm{C}_{28} \mathrm{H}_{20} \mathrm{~S}_{5} \mathrm{ClN}_{9} \mathrm{O}_{16(a c)}^{4-}+89 \mathrm{H}_{2} \mathrm{O}_{2(a c)} \longrightarrow \\
& 28 \mathrm{CO}_{2(g)}+91 \mathrm{H}_{2} \mathrm{O}_{(l)}+9 \mathrm{NO}_{3(a c)}^{-}+5 \mathrm{SO}_{4(a c)}^{=}+\mathrm{Cl}_{(a c)}^{-}+16 \mathrm{H}_{(a c)}^{+}
\end{aligned}
$$

Los ensayos catalíticos a diferentes valores de $\mathrm{pH}$ indicaron que, a $\mathrm{pH}$ ácido $(\mathrm{pH}=3,6)$ el porcentaje de conversión o degradación del colorante es mucho mayor que las conversiones a pH 5,6 y neutro. La posible explicación para este efecto puede estar relacionada con la cercanía de estos valores ( $\mathrm{pH}$ de 5,6 y 7) al punto de carga cero del sólido. En el punto de carga cero, la adsorción de este reactivo disminuye, así como también se ve afectada la trasferencia electrónica para la generación de las especies radicalarias. De otro lado, el porcentaje de conversión del colorante experimentó un incremento al aumentar el flujo de aire (0, 2, 6 y $13 \mathrm{~L} / \mathrm{h})$; esto permite concluir que la degradación del colorante se favorece con el aumento de la cantidad de oxígeno. De acuerdo a la literatura, el oxígeno actúa como agente aceptor de electrones en las reacciones de fotocatálisis, retirando los electrones fotogenerados en la banda de conducción del catalizador y disminuyendo las recombinaciones electrón-hueco (Rauf et al., 2011). En cuanto a la carga de catalizador, se encontró que al aumentar dicha carga de 100 ppm a 200 ppm el porcentaje de degradación del colorante también aumentó, como producto de una mayor cantidad de sitios activos. Sin embargo, al incrementar la carga de catalizador a 300 y 400 ppm, la conversión disminuyó considerablemente. Este comportamiento es comúnmente asociado al exceso de partículas sólidas, el cual reduce la penetración de la radiación en el medio de reacción (Gaya y Abdullah, 2008; Rauf et al., 2011).

Teniendo en cuenta los resultados anteriores de optimización, la evaluación del desempeño fotocatalítico de todos los sólidos se realizó a $\mathrm{pH}=3,6$; la carga de catalizador fue de $200 \mathrm{ppm}$ y un flujo de aire seco de $13 \mathrm{~L} . \mathrm{h}^{-1}$. El resto de condiciones se describieron en la parte experimental. Adicionalmente, se realizaron ensayos de control (con catalizador y lámpara apagada, y sin catalizador y lámpara encendida). La evaluación fotacatalítica de todos los sólidos sintetizados mostró porcentajes de degradación del colorante AR145 mayores o iguales a $85 \%$ en sólo seis horas. En la Figura 4 se muestran (de manera representativa) las curvas de degradación fotocatalítica del colorante AR145, empleando algunos de los sólidos sintetizados. También se comparan dichas curvas con la obtenida para el fotocatalizador comercial $\mathrm{TiO}_{2}$-Evonik. La degradación catalítica del colorante AR145 mediante los sólidos sintetizados es casi comparable con la del $\mathrm{TiO}_{2}$ de referencia. Sin embargo, la menor actividad fotocatalítica de los sólidos sintetizados se atribuye a la ausencia de pequeñas cantidades de la fase rutilo. Se sabe que, la adición de cantidades menores de rutilo (alrededor del 20\%) eleva la actividad fotocatalítica de la fase de anatasa. Como se pudo observar en los resultados de difracción de rayos X (Figura 3), anatasa fue la única fase detectada en los sólidos sintetizados, mientras que el $\mathrm{TiO}_{2}$-Evonik de referencia muestra pequeñas cantidades de la fase rutilo. Estos resultados ponen en evidencia la potencialidad de los sólidos obtenidos de un mineral natural (ilmenita) como fotocatalizadores eficientes para la degradación del colorante AR145.

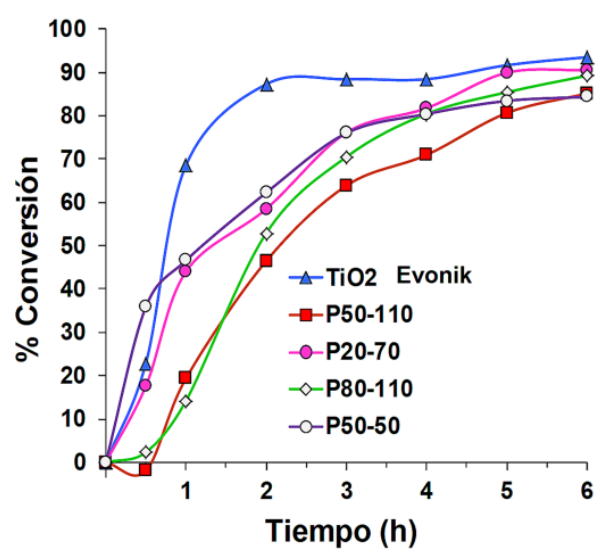

Figura 4. Curvas de degradación fotocatalítica del colorante amarillo reactivo 145 (AR145) usando los sólidos $\mathrm{Fe}(\mathrm{III})-\mathrm{TiO}_{2}$ sintetizados como fotocatalizadores. Los resultados se comparan con la actividad fotocatalítica del $\mathrm{TiO}_{2}$ comercial (Evonik P25).

Por otra parte, la Figura 5 muestra los espectros IR de los sólidos después de ser usados en la degradación del colorante, extraídos por filtración al final de 6 horas de reacción, secados en estufa, sin ser lavados. Con ello se evidencia la ausencia de materia orgánica adsorbida en los sólidos (no se presentan vibraciones relacionadas con estiramientos o flexiones de enlaces tipo C-C, anillos aromáticos o enlaces $\mathrm{N}-\mathrm{H}$, entre otros, característicos del colorante AR145). Dado que el perfil de IR para los catalizadores evaluados al final de la reacción es igual al encontrado en los sólidos antes de ser usados en la reacción, se puede concluir que la decoloración de las soluciones de AR145, muy probablemente, corresponde a la reacción de oxidación completa de dicho contaminante.

En la Figura 5 se indican las bandas de absorción características del $\mathrm{TiO}_{2}$. La banda pequeña situada en 1200 $\mathrm{cm}^{-1}$ corresponde a vibraciones Ti-O-Ti (Ganesh et al., 2012) y la banda ancha entre 400 y $800 \mathrm{~cm}^{-1}$ se atribuye vibraciones del $\mathrm{TiO}_{2}$ o $\mathrm{Fe}(\mathrm{III})-\mathrm{TiO}_{2}$ (Navío et al., 1999; Torres-Luna et al., 2014). Alrededor de $3400 \mathrm{~cm}^{-1}$ se observa una banda ancha atribuida a vibraciones de estiramiento de enlaces $\mathrm{O}-\mathrm{H}$ del agua adsorbida, y en $1626 \mathrm{~cm}^{-1}$ se obtienen las flexiones de dichos enlaces (Ganesh et al., 2012; Torres-Luna et al., 2014). 


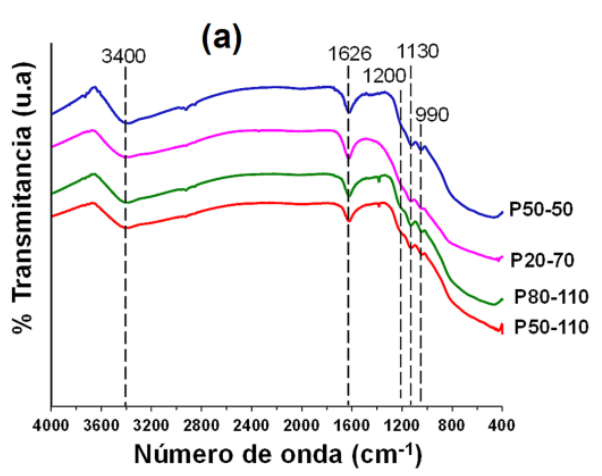

(b)

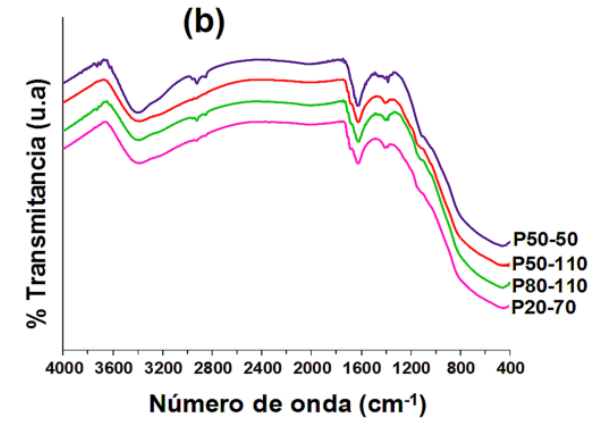

Figura 5. Espectros IR de algunos catalizadores representativos. a) Antes de ser usados en la reacción de degradación fotocatalítica del colorante AR145, y b) después de ser empleados en 6 horas de reacción (secados y sin ser lavados).

\section{CONCLUSIONES |}

Se desarrolló con éxito la degradación fotocatalítica heterogénea del colorante textil amarillo reactivo 145 (AR145), bajo condiciones ambientales, alcanzándose porcentajes de conversión iguales o mayores que $85 \%$ en seis horas de reacción. Para ello se empleó un conjunto de catalizadores sólidos sintetizados a partir del mineral ilmenita, mediante tratamientos ácidos a diferentes valores de temperatura. Los resultados de la caracterización de los sólidos confirmaron la obtención de la fase cristalina anatasa, con ausencia de la fase rutilo, en todos los casos. Los catalizadores sintetizados presentaron mejores propiedades texturales que el $\mathrm{TiO}_{2}$ comercial empleado como referencia. No se observaron diferencias importantes en la actividad catalítica de los sólidos sintetizados, lo que confirma la posibilidad de obtener fotocatalizadores activos mediante condiciones de síntesis muy suaves de temperatura y concentración de ácido.

\section{AGRADECIMIENTOS |}

Los autores agradecen a la Universidad Nacional de Colombia-Bogotá por el apoyo logístico en el desarrollo del presente trabajo. Esta investigación se desarrolló en el Laboratorio de Diseño y Reactividad de Estructuras Sólidas (Lab-DRES), del Departamento de Química de la Universidad Nacional de Colombia, sede Bogotá.

\section{REFERENCIAS}

- Azbar N, Yonar T y Kestioglu K. 2004. Comparison of various advanced oxidation processes and chemical treatment methods for COD and color removal from a polyester and acetate fiber dyeing effluent. Chemosphere, 55: 35-43.

- Bokare AD y Choi W. 2014. Review of iron-free Fenton-like systems for activating $\mathrm{H}_{2} \mathrm{O}_{2}$ in advanced oxidation processes. Journal of Hazardous Materials, 275: 121-135.

- Carriazo JG, Moreno-Forero M, Molina RA y Moreno S. 2010. Incorporation of titanium and titanium-iron species inside a smectite-type mineral for photocatalysis. Applied Clay Science, 50: 401-408.

- Chernet T. 1999. Effect of mineralogy and texture in the $\mathrm{TiO}_{2}$ pigment production process of the Tellnes ilmenite concentrate. Mineralogy and Petrology, 67: 21-32.

- Colpini LMS, Lenzi GG, Urio MB, Kochepka DM y Alves HJ. 2014. Photodiscoloration of textile reactive dyes on $\mathrm{Ni} / \mathrm{TiO}_{2}$ prepared by the impregnation method: Effect of calcination temperature. Journal of Environmental Chemical Engineering, 2: 2365-2371.

- Crediseguro. Informe sector textil y confecciones colombianos. 2010. Medellín, Colombia. Disponible en: http://www.crediseguro.com.co/dmdocuments/INFORME_SECTOR_TEXTIL_ Marzo_2010.pdf

- DAMA. 2004. Guía ambiental para el sector textil, Bogotá, Colombia. Disponible

en:http://www.minambiente.gov.co/Puerta/destacado/vivienda/gestion_ds_ municipal/nuevas guias/TEXTIL.pdf

- De Souza SM, Bonilla KAS y de Souza AAU. 2010. Removal of COD and color from hydrolyzed textile azo dye by combined ozonation and biological treatment. Journal of Hazardous Materials, 179: 35-42.

- Ganesh I, Kumar PP, Gupta AK, Sekhar PSC, Radha K, Padmanabham G y Sundararajan G.. 2012. Preparation and characterization of Fe-doped $\mathrm{TiO}_{2}$ powders for solar light response and photocatalyticapplications. Processing and Application of Ceram. 6 (1), 21-36.

- García-Muñoz P, Pliego G, Zazo JA, Barbero B, Bahamonde A y Casas JA. 2016. Modified ilmenite as catalyst for CWPO-Photoassisted process under LED Light. Chemical Engineering Journal. In Press. DOI:10.1016/j.cej.2016.05.093.

- Gaya UI y Abdullah AH. 2008. Heterogeneous photocatalytic degradation of organic contaminants over titanium dioxide: A review of fundamentals, progress and problems. Journal of Photochemistry and Photobiology C, 9: $1-12$.

- Guimaraes de Oliveira A, Nascimento JP, Gorgulho HF, Martelli PB, Furtado CA, Figueiredo JL. 2016. Electrochemical synthesis of $\mathrm{TiO}_{2} / \mathrm{Graphene}$ oxide composite films for photocatalytic applications. Journal of Alloys and Compounds, 654: 514-522

- Gül \$̧ y Özcan-Yıldırım Ö. 2009. Degradation of Reactive Red 194 and Reactive Yellow 145 azo dyes by $\mathrm{O}_{3}$ and $\mathrm{H}_{2} \mathrm{O}_{2} / \mathrm{UV}-\mathrm{C}$ processes. Chemical Engineering Journal, 155: 684-690.

- Khataee AR. 2009. Photocatalytic removal of C.I. Basic Red 46 on immobilized $\mathrm{TiO}_{2}$ nanoparticles: Artificial neural network modelling. Environmental Technology, 30: 1155-1168.

- Ministerio del Medio Ambiente. 2001. Guía de Buenas Prácticas para el Sector Textil, Colombia. Disponibles en: http://www.minambiente.gov.co/documentos/Textiles.pdf

- Muñoz M, de Pedro ZM, Casas JA y Rodríguez JJ. 2015. Preparation of magnetite-based catalysts and their application in heterogeneous Fenton oxidation - A review. Applied Catalysis B, 176-177: 249-265.

- Navío JA, Colón G, Macías M, Real C y Litter MI. 1999. Iron-doped titania semiconductor powders prepared by a sol-gel method. Part I: synthesis and characterization. Applied Catalysis A, 177:111-120.

- Oller I, Malato S y Sánchez-Pérez JA. 2011. Combination of Advanced Oxidation Processes and biological treatments for wastewater 
decontamination-A review. Science of the Total Environment, 409: 41414166.

- Patil NN y Shukla SR. 2015. Degradation of reactive yellow 145 dye by persulfate usingmicrowave and conventional heating. Journal of Water Process Engineering, 7: 314-327.

- Rauf MA, Meetani MA, Hisaindee S. 2011. An overview on the photocatalytic degradation of azo dyes in the presence of $\mathrm{TiO}_{2}$ doped with selective transition metals. Desalination, 276: 13-27.

- Robinson T, McMullan G, Marchant R y Nigam P. 2001. Remediation of dyes in textile effluent: a critical review on current treatment technologies with a proposed alternative. Bioresource and Technology, 77: 247-255.

- Sasikumar C, Rao DS, Srikanth S, Ravikumar B, Mukhopadhyay NK y Mehrotra SP. 2004. Effect of mechanical activation on the kinetics of sulfuric acid leaching of beach sand ilmenite from Orissa, India. Hydrometallurgy, 75: 189-204.

- Sathishkumar P, Anandan S, Maruthamuthu P, Swaminathan T, Zhou M y Ashokkumar M. 2011. Synthesis of $\mathrm{Fe}^{3+}$ doped $\mathrm{TiO}_{2}$ photocatalysts for the visible assisted degradation of an azo dye. Colloids and Surfaces A: Physicochemical Engineering Aspects, 375: 231-236.
- Teoh WY, Amal R, Mädler L y Pratsinis SE. 2007. Flame sprayed visible lightactive $\mathrm{Fe}-\mathrm{TiO}_{2}$ for photomineralisation of oxalic acid. Catalysis Today, 120: 203-213.

- Torres-Luna JA, Carriazo JG y Sanabria NR. 2014. Efecto de la temperatura de calcinación en las propiedades texturales y estructurales de $\mathrm{Fe}(\mathrm{III})-\mathrm{TiO}_{2}$. Revista Facultad de Ciencias Básicas, 10: 186-195.

- Torres-Luna JA, Carriazo JG y Sanabria NR. 2016. Delaminated montmorillonite with iron(III)- $-\mathrm{TiO}_{2}$ species as a photocatalyst for removal of a textile azo-dye from aqueous solution. Environmental Technology, 37: 1346-1356.

- Verma AK, Dash RR y Bhunia P. 2012. A review on chemical coagulation/flocculation technologies for removal of colour from textile wastewaters. Journal of Environmental Management, 93: 154-168.

- Zaharia C y Suteu D. 2012.Textile Organic Dyes - Characteristics, Polluting Effects and Separation/Elimination Procedures from Industrial Effluents - A Critical Overview. En: Puzyn $T$ y Mostrag-Szlichtyng A (Eds.). Organic Pollutants Ten Years after the Stockholm Convention - Environmental and Analytical Update, INTECH, Rijeka, Croatia, pp. 55-86.

- Zhang X, Zhou M y Lei L. 2006. Co-deposition of photocatalytic Fe doped $\mathrm{TiO}_{2}$ coatings by MOCVD. Catalysis Communication, 7: 427-431. 